\title{
Oratoria y relaciones públicas: las Filípicas y las Olínticas de Demóstenes como una de las primeras campañas de comunicación institucional
}

\author{
Oratory and public relations: Demosthenes's Philippics and \\ Olynthiacs like one of the first campaigns of institutional \\ communication.
}

\author{
Marta Pulido Polo ${ }^{1}$ \\ Universidad de Sevilla \\ martapulido@us.es
}

Recepción: 22.11.2016 Revisión: 27.12.2016 Aceptación: 27.12.2016 Publicación: 15.01.2017

\begin{abstract}
Resumen
En el siglo IV a. C. comienza el declive de la supremacía ateniense en Grecia derivado de las continuas luchas internas, el auge del imperio persa y la amenaza militar de una Macedonia fuerte conducida por Filipo II. En este contexto Demóstenes desarrollará, a través de sus Filípicas y sus Olínticas, la que puede ser considerada una de las primeras campañas de comunicación y relaciones públicas institucionales de la antigüedad, sustentada en la persuasión de la oratoria, para concienciar a la opinión pública acerca de los peligros que el régimen autocrático macedonio representaba para los principios democráticos atenienses. $A$ través de una revisión teórica fundamentada en el uso de fuentes secundarias, sobre todo bibliográficas, los resultados de este trabajo demuestran que la campaña desarrollada por Demóstenes contra Filipo II de Macedonia puede ser considerada como una de las primeras campañas estratégicas de relaciones públicas que, con una finalidad persuasiva, se sustentó en el uso intencional de la oratoria.
\end{abstract}

Palabras clave: Relaciones públicas institucionales, Demóstenes, Filípicas, Olínticas, Oratoria.

\footnotetext{
${ }^{1}$ Consultora de Relaciones Públicas. Profesora Acreditada Contratada Doctora, trabaja como Asociada en la Universidad de Sevilla. Doctora por la Universidad de Sevilla. Técnico en Comunicación Empresarial y Protocolo. Directora de la Consultora la bicicleta de Design \& Communication. Directora de Comunicación de la Organización Internacional de Ceremonial y Protocolo (OICP). Presidenta de la Asociación de Relaciones Públicas y Protocolo de Andalucía (ARPPA). Miembro del Grupo de investigación en Relaciones Públicas, Ceremonial y Protocolo LAUREA Hispalis. Miembro del Grupo de Investigación Historia del Pensamiento Jurídico-Político (GIHPJ-P). martapulido@us.es.
} 


\begin{abstract}
In the 4th century B.C. it begins the decline of the Athenian supremacy in Greece derived from the continuous internal fights, the summit of the Persian Empire and the military threat of a strong Macedonia led by Philip II. In this context Demosthenes will develop, through its Philippics and Olynthiacs, one of the first public relations campaigns of the antiquity, sustained in the persuasion of the oratory, to report to the public opinion about the danger that the autocratic Macedonian regime was representing by the democracy of Athens. Across a theoretical review based on the use of secondary sources, especially bibliographical, the results of this work demonstrate that the campaign developed by Demosthenes against Philip II of Macedonia can be considered to be one of the first strategic campaigns of public relations that, with a persuasive purpose, were sustained in the intentional use of the oratory.
\end{abstract}

Keywords: Institutional public relations, Demosthenes, Philippics, Olynthiacs.

\title{
Sumario
}

1. Introducción

2. Metodología y objetivos

3. Contextualización histórica

4. Demóstenes y la oratoria

5. Discusión de los resultados: las Filípicas y las Olínticas de Demóstenes, la comunicación institucional y la gestión de la opinión pública

6. Conclusiones

7. Referencias bibliográficas.

\section{Summary}

1. Introduction

2. Methodology and objectives

3. Historical context

4. Demosthenes and the oratory

5. Discussion of the results: Demosthenes's Philippics and Olynthiacs, the institutional communication and the management of the public opinion

6. Conclusions

7. Bibliographical references

\section{INTRODUCCIÓN}

Para XIFRA (2011:44), la campaña de opinión pública que Demóstenes sostiene y desarrolla contra la expansión de Filipo II de Macedonia y a favor de la supremacía y la democracia atenienses, puede ser considerada una de las primeras campañas de relaciones públicas de la antigüedad. La relevancia de este texto radica, por tanto, en reivindicar el origen histórico de lo que hoy denominamos comunicación institucional, tanto en las Olínticas como en las Filípicas de DEMÓSTENES (1998). 
La dirección de la comunicación institucional se materializa fundamentalmente a través de la gestión de las relaciones que se establecen entre una organización y los públicos de su entorno, tal y como describe la perspectiva relacional de las relaciones públicas propugnada inicialmente por LEDINGHAM Y BRUNNING (1998) y desarrollada posteriormente por LEDINGHAM (2003). Los supuestos desarrollados por LEDINGHAM (2006) proponen una reconceptualización de la gestión de la comunicación en empresas e instituciones reposicionando el eje principal sobre el que pivota la actividad relacionista desde la comunicación hasta el concepto de relación.

Desde esta perspectiva, la perspectiva relacional de las relaciones públicas defiende que la actividad principal del relacionista público a la hora de gestionar la comunicación organizacional radica en el saber administrar adecuadamente el entramado relacional que necesariamente se gesta entre una organización y sus públicos clave o stakeholders, es decir, de aquellos sectores del entorno de los que depende su supervivencia exitosa como organización, o su fracaso. Bajo este enfoque relacional, la adecuada gestión de las relaciones organización-públicos, institución-públicos o empresa-públicos tiene una consecuencia principal sobre la teoría de la comunicación de las instituciones: la actividad principal de la gestión de la comunicación se basa en la gestión de las relaciones y no en la gestión de la comunicación. Si bien es cierto que, a la hora de planificar de forma estratégica el entramado relacional institucional, se utilizan, de forma auxiliar y subsidiaria, técnicas tradicionalmente ligadas al ámbito comunicacional tales como las notas de prensa, la organización de actos, el mecenazgo, o las alocuciones en foro de interés.

En este contexto, parece evidente que, desde las primeras manifestaciones de vida en comunidad, el ser humano fue desarrollando los principios básicos de la persuasión a través del empleo intencionado de la palabra hablada y los discursos, dando lugar al progresivo desarrollo del concepto de oratoria, concebida como una disciplina orientada a persuadir y situar, a través de la declamación de discursos, convenientes mensajes institucionales en la opinión pública de cada momento histórico. De hecho, en numerosas culturas antiguas, la oratoria formaba parte del adiestramiento y formación de aquellos segmentos poblacionales que pertenecían a la esfera del poder y que estaban llamados a continuar y perpetuar la élite política y social de su comunidad.

Desde este punto de vista, la oratoria podría considerarse, bajo los parámetros actuales, una técnica específica de relaciones públicas encaminada a situar, de forma creíble, el punto de vista de la organización ante una opinión pública determinada a través de la utilización de la palabra hablada con una finalidad informativa y argumentativa de consecuencias persuasivas.

\section{METODOLOGÍA Y OBJETIVOS}

Este trabajo nace como una oportunidad para observar las Filípicas y las Olínticas de Demóstenes como una de los primeros ejercicios persuasivos de la antigüedad, orquestados de forma estratégica, para situar de forma intencionada y consciente un determinado mensaje institucional ante la opinión pública ateniense del momento. El objetivo principal de este trabajo es, por tanto, describir y analizar de forma global estos siete discursos de Demóstenes como una de las primeras campañas de relaciones públicas institucionales 
orientada a fomentar la supremacía y los valores democráticos que conformaban la cosmovisión de la Grecia clásica.

Para desarrollar este objetivo principal es necesario cubrir los siguientes objetivos secundarios:

OS1: Contextualizar desde un punto de vista socio-político e histórico las Filípicas y las Olínticas de Demóstenes

OS2: Introducir la biografía de Demóstenes en relación con el desarrollo y empleo de la oratoria y la persuasión orientada a situar determinados mensajes institucionales en la opinión pública.

OS3: Analizar las Filípicas y las Olínticas desde la perspectiva de las relaciones públicas institucionales, como posible precursor de las campañas actuales de gestión de la comunicación institucional.

Para alcanzar estos objetivos, se realiza un diseño metodológico sustentado en el uso de fuentes de datos secundarios, sobre todo bibliográficos, en torno a las relaciones públicas, Demóstenes, la oratoria y la persuasión de masas.

\section{CONTEXTUALIZACIÓN HISTÓRICA}

Después de la Guerra del Peloponeso, en el siglo IV a. C., comienza a resquebrajarse la supremacía de Atenas en el mundo griego por la presencia de guerras internas, el inminente peligro del aumento del poder de Persia, y el ingente avance militar de una Macedonia capitaneada por Filipo II. En este contexto, Isócrates dirigió un discurso a Filipo II proponiéndole sostener el poder político de Grecia con Atenas al mando, mientras que Demóstenes se centró en desarrollar una campaña de opinión pública impulsando a sus compatriotas a oponerse a la amenaza que representaba, para la democracia ateniense, Filipo II de Macedonia.

Tal y como afirma NIETO ORRIOLS (2013: 39):

En el siglo IV a. de C., luego de la Guerra del Peloponeso y en el marco del desmembramiento de Atenas como líder del mundo griego, la política ateniense se vio afectada internamente por la presencia de guerras fratricidas, promovidas por los intereses individualistas de sus dirigentes. En este contexto $y$ ante el inminente peligro del aumento del poder de Persia, del avance militar de Macedonia y el conflicto interno de la polis, Isócrates dirigió un discurso a Filipo II proponiéndole sostener el poder político de Grecia con Atenas al mando. Su propuesta panhelénica consistía en unificar el poder político a través de un individuo que ostentara las cualidades morales propicias para llevar a Grecia a un nuevo apogeo cultural $y$, en ese contexto, enfrentar al Imperio Persa, enemigo continuado de Grecia. Por su parte, Demóstenes manifestó una postura contraria, argumentando que las opiniones a favor de Filipo eran erróneas, pues el macedonio era un bárbaro inconfiable cuyas intenciones eran 
someter a Grecia bajo su yugo tiránico, por lo que sugiere declararle la guerra.

De esta manera, con los cuatro discursos que pronuncia contra Filipo II, que pasarán a la historia conocidos como las Filípicas, y los tres discursos en defensa del pueblo de Olinto, conocidos como las Olínticas, Demóstenes pretende transformar la voluntad de su pueblo ante la nueva situación que amenazaba Atenas, consagrándose como un político y un orador de altura y uno de los antecedentes más reputados de los relacionistas públicos actuales.

\section{DEMÓSTENES Y LA ORATORIA}

Las graves circunstancias familiares que le toca vivir, obligan a Demóstenes (Atenas, 384 aC Calauria, $322 \mathrm{aC}$ ) a adiestrarse en las artes de la oratoria desde temprana edad. Su padre, que fallece cuando Demóstenes tiene 7 años de edad, le deja una fortuna como herencia que es custodiada bajo fideicomiso. Al alcanzar la mayoría de edad, Demóstenes, se ve obligado a reclamar judicialmente la herencia que sus desleales tutores se habían apropiado de forma ilegal, aunque solo consigue recuperar una parte. A partir de entonces se dedica a escribir discursos para litigios legales particulares, aunque poco a poco comienza a interesarse por la vida pública y la política ateniense. Paradójicamente, a pesar de pasar a la historia como uno de los grandes oradores clásicos, sus biógrafos describen a un Demóstenes con problemas de habla (tartamudez), que trataba de paliar con duros ejercicios de declamación entre los que, al parecer, se encontraban la práctica de hablar con guijarros en la boca² o declamar al viento en la orilla del mar.

De hecho, tal y como representan numerosas obras pictóricas, la historia nos muestra a un Demóstenes que dedicaba largas horas de ensayo y entrenamiento a la búsqueda de una combinación enfática de los elementos verbales y no verbales para imprimir mayor eficacia persuasiva a sus alocuciones públicas. En concreto, destaca la obra de 1859 de Delacroix Demóstenes en la costa, que recrea la imagen de un Demóstenes que, al parecer, gustaba de ensayar sus discursos ante el ruido de las olas del mar para fortalecer el tono de su voz y modular adecuadamente el sonido declamando al viento. La imagen destaca el cultivo, con tintes persuasivos, de los principios no verbales en las alocuciones públicas tales como la gestualidad, el tono de la voz, o la dicción.

Cabe destacar que, en la retórica griega, al igual que sucediera posteriormente en la cultura romana, la oratoria judicial gozaba de una posición de liderazgo (TELLEGEN-COUPERUS, 2003) cuestión esta que posiblemente propicia que Demóstenes muestre tal interés por profundizar en el estudio y la práctica de la oratoria con claras finalidades persuasivas.

\section{LAS FILÍPICAS Y LAS OLÍNTICAS DE DEMÓSTENES}

Tal y como hemos adelantado en párrafos precedentes, Demóstenes dedica gran parte de su vida a luchar contra la expansión del reino de Macedonia y por reforzar los valores democráticos atenienses, impulsando a sus compatriotas a oponerse a la amenaza que representaba el ánimo conquistador y expansivo que representaban las incursiones de Filipo

\footnotetext{
${ }^{2}$ http://www.epdlp.com/escritor.php?id=7324
} 
II de Macedonia. Este esfuerzo persuasivo quedará plasmado como un ejemplo de gestión de comunicación institucional de la política ateniense, tanto a través de sus Filípicas como de sus Olínticas. Las Filípicas aluden a cuatro discursos pronunciados por Demóstenes contra Filipo II: la primera versa sobre la paz; la segunda, acerca de una carta misiva de Filipo; la tercera trata de los sucesos del Quersoneso y la cuarta, sobre la declaración de guerra de Filipo (CORREDOR TAPIAS, 2012: 138).

En su Primera Filípica (351 a.C.), Demóstenes presagia ya las intenciones conquistadoras de Filipo de Macedonia puesto que solo dos años más tarde, el macedonio ataca y conquista Olinto, aliada de Atenas. A través de tres discursos, conocidos como las Olínticas, Demóstenes exhorta a los atenienses a la necesidad de ayudar a sus aliados contra la amenaza macedonia. Cuando Olinto es destruido, Demóstenes dedicaría los ocho años siguientes a insistir y tratar de advertir a los atenienses, a través del resto de sus Filípicas, sobre el peligro del advenimiento macedonio ${ }^{3}$.

En cualquier caso, cabe reseñar que tanto las Olínticas como las Filípicas retratan a un Filipo tiránico, un bárbaro destinado a ser súbdito de los griegos, y nunca su líder. Es decir, Demóstenes utiliza sus discursos como una poderosa arma de persuasión de masas comparable a las técnicas propagandísticas que, siglos más tarde, se desarrollarían fundamentalmente en torno a la I y II Guerra Mundial. Tal y como afirma SANCHO ROCHER (2002: 245):

Los tópicos utilizados, los principios elegidos para la argumentación y la descripción de sus rivales beben de un caudal de ideas popular y, casi sin duda, democrático. Puesto que en su época era obligado para cualquier político activo mostrarse ante la audiencia como el mayor defensor de los derechos populares, la única evidencia a favor de la solidez de sus principios es la actitud mantenida por Demóstenes tras el 338 y hasta su muerte. En todo caso, el carácter de sus escritos es muy diferente al de los de Isócrates, mucho más perentorio y directo; su objetivo no es convencer o educar a una élite sino mover a las masas a la decisión o a la actuación.

En concreto, en la Segunda Olíntica, Demóstenes intentaba promover un cambio volitivo entre sus conciudadanos, incitándolos a pasar a la acción contra Filipo. Para ello utiliza un argumento orientado a desprestigiar el carisma y el poder del líder ante sus propios administrados, argumento que seguimos viendo en la actualidad en las campañas electorales nacionales e internacionales, tratando así de posicionar un mensaje en la opinión pública ateniense que cuestionaba la autoridad pragmática del Rey macedonio ante los súbditos de su propio país. (...) Esta idea, sincera o no, radica en la imagen tradicional de la soledad del tirano, rodeado de opositores internos. Los pueblos del entorno macedonio, los primeros en caer bajo su férula, también deberían alzarse ante el tirano ya que, según Demóstenes, no estaban acostumbrados a obedecer (...) Demóstenes anima constantemente a desconfiar de tal personaje dada la incompatibilidad entre los dos sistemas de democracia y monarquía (SANCHO ROCHER, 2002: 249-250).

\footnotetext{
${ }^{3}$ http://www.epdlp.com/escritor.php?id=7324
} 


\section{DISCUSIÓN DE LOS RESULTADOS: LAS FILÍPICAS DE DEMÓSTENES, LA COMUNICACIÓN INSTITUCIONAL Y LA GESTIÓN DE LA OPINIÓN PÚBLICA}

Aunque el origen de las relaciones públicas, tal y como las conocemos en la actualidad, se remonta al siglo XIX y principios del XX con el surgimiento de la publicity de la mano de las primeras figuras históricas de la disciplina como Barnum, Ivy Lee $y$, posteriormente, Bernays, lo cierto es que, a lo largo de la historia del ser humano, se han venido desarrollando estrategias orientadas a generar, en las sociedades de cada época, mensajes que legitiman a quienes ocupan la esfera del poder bien político, bien religioso. De hecho, no es extraño encontrar diversas manifestaciones artísticas o rituales a través de las cuales aquellas incipientes organizaciones sociales rendían culto a determinados dioses o líderes. Estas manifestaciones legitimantes obedecían, además, a una naturaleza estratégica y a una finalidad persuasiva que funcionaban de forma armónica a un doble nivel: el endógeno y el exógeno. A nivel endógeno porque, a nivel interno, para la supervivencia de la comunidad era importante tener un líder fuerte bien posicionado dentro del propio grupo que impusiera orden y respeto en previsión de posibles golpes de estado o rebeliones internas. $A$ nivel exógeno porque, a nivel externo, convenía reforzar la imagen de un líder fuerte, arropado por su propio pueblo, al que era difícil de derrocar, en previsión de posibles invasiones.

En este contexto, parecen evidentes las claras similitudes entre aquellas primigenias campañas de opinión pública de la antigüedad y los actuales programas estratégicos de relaciones públicas.

\section{CONCLUSIONES}

Las relaciones públicas describen un proceso directivo a través del cual las organizaciones privadas y las instituciones públicas tratan de establecer y gestionar un sistema de interrelaciones mutuamente beneficiosas con aquellos públicos de los que depende su éxito o fracaso (CUTLIP, CENTER y BROOM, 2001:37) con la finalidad de encontrar un equilibrio de intereses organización-públicos.

Este proceso implica, necesariamente, el establecimiento de un sistema de comunicación bidireccional y dialógico (GRUNIG, 2000) en el que la organización sea capaz de interpretar las necesidades y expectativas de sus stakeholders del mismo modo que sus públicos puedan entender la misión y los valores de la organización (SEITEL, 2002). Bajo estas premisas, la acción planificada de las relaciones públicas desde los postulados del pensamiento estratégico, debe encaminarse a situar en la opinión pública organizacional, un mensaje institucional determinado que debe caracterizarse por su naturaleza informativa y su finalidad persuasiva (XIFRA, 2003).

Para AMORÓS PONS y COMESAÑA COMESAÑA (2012: 119):

La Dirección de Comunicación es la responsable de planificar el uso de los diversos medios de comunicación tanto internos como externos, con la finalidad de comunicar a los distintos públicos de la comunidad los mensajes que una empresa o institución busca promover, con el objetivo 
de generar una imagen institucional positiva, cuando comunica las acciones, eventos o información que genera a través de múltiples acciones de comunicación.

De este modo, aquellos esfuerzos persuasivos de Demóstenes, a través de la oratoria, por situar en la opinión pública ateniense un mensaje institucional alertando a cerca de los peligros que representaban la autocracia y el poder ilimitado de la monarquía macedonia (PINA POLO, 1993: 179) para los principios democráticos representados por Atenas, no pueden sino implicar, bajo el prisma del siglo XXI, una concepción estratégica de las relaciones con los públicos que puede enmarcarse en el modelo de la co-orientación para la búsqueda del consenso (AUSTIN Y PINKLETON, 2015: 64).

En la actualidad, la finalidad persuasiva de las relaciones públicas, resulta un elemento vertebrante e inherente de la actividad de la dirección de la comunicación en las organizaciones y de las relaciones públicas, puesto que, del mismo modo que Demóstenes a través de la oratoria, las relaciones públicas utilizan la argumentación y la información como elementos clave para engrasar el sistema de comunicación de doble direccionalidad, a la que ya aludía BERNAYS (1998) en los comienzos de las primeras teorizaciones relacionistas, y mediante la cual el público es capaz de interpretar a la organización y la organización de interpretar a sus públicos.

Tal y como afirma BOTERO ESCOBAR (2012: 135):

Cuando buscamos establecer una relación entre la teoría de la comunicación y las relaciones públicas, es obligación teórica aclarar que la primera abarca a la segunda; es decir, las relaciones públicas como técnica y disciplina encuentran en la comunicación su principal instrumento para desarrollarse, para convertirse en realidad. El uso de los medios de comunicación y de las herramientas de persuasión fundamentadas en el lenguaje oral y escrito ha hecho de las relaciones públicas una disciplina de amplio reconocimiento en Estados Unidos y Europa, configurando una profesión de alto lucro y de consolidada posición social.

Utilizadas de forma estratégica, las relaciones públicas coadyuvan a las organizaciones a situar, en la agenda mediática y en la opinión pública mensajes corporativos, que fomentan las relaciones mutuamente beneficiosas organización-públicos del entorno y la búsqueda de un punto de interés neutral, generando un espacio para el consenso y el entendimiento y, de este modo, fomentar el desarrollo de un clima agradable donde la organización y su entorno puedan, mutuamente, desarrollar sus metas organizativas o individuales.

Desde esta perspectiva, la influencia de los medios de comunicación de masas como intermediarios necesarios entre la vida corporativa e institucional y el posicionamiento de determinados mensajes de legitimación en la opinión pública de un entorno organizacional cada vez más complejo es altamente reseñable. Tal y como destaca CASTILLO ESPARCIA (2011):

En este sentido, el papel de los medios de comunicación se decanta hacia dos tendencias: hacia la concentración e incremento de la diversificación. 
Ese fenómeno señalado en el libro se ha ido incrementado en las últimas décadas con una mayor concentración de los medios y los intentos de los poderes nacionales e internacionales por regular los procesos de concentración.

Disponer de los medios de comunicación significa, en la actualidad, poseer un poder social, no tanto por el contenido que transmiten sino por el ambiente que crea, la atención y el mimetismo que despierta. El medio actúa como un espejo que refleja la realidad social y, al mismo tiempo, es el lugar en el que se crea ésta.

La intensa presencia de los medios de comunicación y su influencia ha posibilitado que se profiera mucho sobre su poder y de las funciones y posiciones que ocupan en la dinámica política. Desde definidos como cuarto poder hasta contrapoder, pasando por la influencia que pueden ejercer sobre los decisores públicos como sujetos que manifiestan públicamente las opiniones de los ciudadanos, los mass media son el centro informativo de una sociedad cada vez más compleja.

En este contexto, las antiguas estrategias derivadas de la oratoria clásica se estudian en la actualidad aplicándose a las fórmulas que las actuales consultoras de comunicación, a nivel nacional e internacional, aplican a la hora de gestionar la formación de portavoces corporativos o institucionales, siendo las alocuciones en foros de interés o el diseño de discursos públicos uno de los recursos más habituales para gestionar las relaciones con el entorno, erigiéndose así, en una técnica transversal de relaciones públicas (XIFRA, 2007) de uso extendido.

\section{REFERENCIAS BIBLIOGRÁFICAS}

AMORÓS PONS, A. Y COMESAÑA COMESAÑA, P., "Las Relaciones Públicas como estrategia de comunicación en los eventos cinematográficos: los Premios Goya", Revista Internacional de Relaciones Públicas, no 3, 2012.

AUSTIN, E. W. Y PINKLETON, B. E., Strategic Public Relations Management: Planning and Managing Effective Communication Programs, New York, Taylor \& Francis, 2015.

BERNAYS, E. L., Cristalizando la opinión pública, Barcelona, Gestión 2000, 1998.

BOTERO ESCOBAR, N. E., "La teoría de la comunicación, las relaciones públicas y la investigación: una relación simbiótica”, Anagramas, nN 21, 2012.

CASTILLO ESPARCIA, A., "Los medios de comunicación como actores sociales y políticos. Poder, medios de comunicación y sociedad", Razón y palabra, no 75, 2011.

http://www.razonypalabra.org.mx/N/N75/monotematico_75/12_Castillo_M75.pdf

CORREDOR TAPIAS, J., "Doce magnos oradores: Breves consideraciones biográficas e intento de acercamiento a uno de sus discursos", Cuadernos de Lingüística Hispánica, no 19, 2012. 
CUTLIP, S. M. Y CENTER, A. H., Relaciones públicas eficaces. Barcelona, Gestión 2000, 2001.

DEMÓSTENES, Las Filípicas. Sobre la corona, Madrid, Ediciones Cátedra, 1998.

GRUNIG, J. E. Y HUNT, T., Dirección de relaciones públicas, Barcelona, Gestión 2000, 2000.

LEDINGHAM, J. A. Y BRUNNING, S. D., "Relationship management in public relations: dimensions of an organization-public relationship" en Public Relations Review, Vol. 24, no 1, 1998.

LEDINGHAM, J. A., "Explicating Relationship Management as a General Theory of Public Relations", Journal of public relations research, no 15, 2003.

LEDINGHAM, J. A., "Relationship management: a general theory of public relations". Public Relations Theory II, Lawrence Erlbaum Associates, Inc. Mahwah, New Jersey, 2006.

NIETO ORRIOLS, D., "La utilidad de la guerra en los discursos de Isócrates y Demóstenes. Derecho, cohesión social y legitimación cultural en Atenas", Intus-Legere Historia, no 1, 2013.

PINA POLO, F., "El ascenso y la hegemonía de macedonia: características del régimen monárquico". POLIS Revista de ideas y formas políticas de la Antigüedad Clásica, no 5, 1993.

SANCHO ROCHER, L., “Las fronteras de la política”, Gerión, no 1, 2002.

SEITEL, F., Teoría y práctica de las relaciones públicas, Madrid, Prentice Hall, 2002.

TELLEGEN-COUPERUS, O., "Retórica y derecho romano", Montegudo, no 8, 2003.

XIFRA, J., Teoría y estructura de las relaciones públicas, Madrid, Mc Graw Hill, 2003.

XIFRA, J., Manual de relaciones públicas e institucionales, Madrid, Tecnos, 2011. 